\title{
Comparative Gene Mapping as a Tool to Understand the Evolution of Pest Crop Insect Chromosomes
}

\author{
Mauro Mandrioli (1), Giada Zambonini and Gian Carlo Manicardi * \\ Department of Life Sciences, University of Modena and Reggio Emilia, Modena 41125, Italy; \\ mauro.mandrioli@unimore.it (M.M.); giadazambonini@hotmail.it (G.Z.) \\ * Correspondence: manicardi.giancarlo@unimore.it; Tel.: +39-0522-52-2059
}

Received: 8 August 2017; Accepted: 5 September 2017; Published: 7 September 2017

\begin{abstract}
The extent of the conservation of synteny and gene order in aphids has been previously investigated only by comparing a small subset of linkage groups between the pea aphid Acyrthosiphon pisum and a few other aphid species. Here we compared the localization of eight A. pisum scaffolds (covering more than $5 \mathrm{Mb}$ and 83 genes) in respect to the Drosophila melanogaster Muller elements identifying orthologous loci spanning all the four A. pisum chromosomes. Comparison of the genetic maps revealed a conserved synteny across different loci suggesting that the study of the fruit fly Muller elements could favour the identification of chromosomal markers useful for the study of chromosomal rearrangements in aphids. A. pisum is the first aphid species to have its genome sequenced and the finding that there are several chromosomal regions in synteny between Diptera and Hemiptera indicates that the genomic tools developed in A. pisum will be broadly useful not only for the study of other aphids but also for other insect species.
\end{abstract}

Keywords: aphid chromosomes; Muller elements; chromosomal rearrangements; synteny

\section{Introduction}

A large number of insect genomes have been wholly sequenced in the last decades in order to better understand their biology and, in particular for pest crop insects, to identify genes that could represent a potential target for their control in the field [1-6].

Insects are essential to maintaining agricultural ecosystems, but some of them are pests that damage $>30 \%$ of agricultural, forestry, and livestock production and cause billions in economic losses annually. Currently, the genomes of at least 140 insects have been sequenced and deposited in public databases and the availability of insect genomes and transcriptomes provided valuable resources for entomological research [1-6]. Indeed, insect genomics allowed the gain of knowledge in several fields, such as functional genomics, comparative analysis of genomic contents and their organization, as well as functional analyses of critical parameters as their capacity to transmit disease agents. A better understanding of many individual genes and gene families has been obtained as well [1-6]. However, most of these projects (except Diptera) completely lacked any information about the chromosomal localization of the identified genes and, as a consequence, the involvement of chromosomal rearrangements in insect biology has been almost neglected.

Data concerning the chromosomal localization of the annotated genes could be, for instance, extremely relevant to understanding the evolution of the sex chromosomes and the sex determining system, which is a topic of great interest for pest crop insects [7,8].

The genome mapping in Diptera evidenced that in Drosophila species six different chromosome arms, the so-called "Muller elements," constitute the building blocks for all Drosophila species. The conservation of the Muller elements extends far beyond Drosophila to, at least, tephritid fruit flies, thought to have diverged from drosophilids 60-70 million years ago, favouring the understanding of 
the mechanisms that shaped the evolution of the dipteran karyotype [9-15]. For instance, chromosomal arms exhibit significant remnants of homology between D. melanogaster and Anopheles gambiae, despite the fact they diverged about 250 million years ago, and about $34 \%$ of their genes colocalize in "microsyntenic" clusters [10].

The genome of the aphid Acyrthosiphon pisum has been published in 2010 [16] and it favoured a better understanding of the biology of Hemiptera, a taxon consisting of a large number of pest crops species $[17,18]$.

Few studies have been focused on the construction of genetic maps in aphids [19]. The first densest pea aphid genetic map has been developed by Hawthorne and Via [20] with the aim to study the aphid host plant specialization. They developed a linkage map of 173 dominant amplified fragment length polymorphism (AFLP) markers grouped into four linkage groups. Successively, Braendle et al. [21] developed an additional seven AFLP markers on the $\mathrm{X}$ chromosome.

From a cytogenetic point of view, aphid chromosomes have been studied mainly in order to identify cytogenetic markers that could be useful for taxonomic identification, as well as for the analysis of karyotype evolution [22-27]. At this time, few genes have been located on chromosomes in aphids $[28,29]$.

In order to improve our knowledge about the gene distribution on aphid chromosomes and to suggest a strategy for the identification of chromosomal markers, here we compared the localization and composition of eight scaffolds (spanning $5.3 \mathrm{Mb}$ and 83 genes) identified in A. pisum in respect to the fruit fly Muller elements. This approach allowed the identification of orthologous loci spanning all the four A. pisum chromosomes. In view of the suggestion that $A$. pisum shows a substantial synteny (together with conserved gene order and orientation) with other Aphidinae [30,31], our approach could be useful to extend genomic information from A. pisum to other aphid species. Lastly, comparative mapping can facilitate not only the investigation of specific evolutionary questions, but also the study of synteny at genomic scales to elucidate chromosome homology, providing a framework for predicting the location of genes in other species, including insects of agricultural interest.

\section{Results}

In order to compare the localization of genes between the A. pisum genome and the D. melanogaster Muller elements, we identified a set of $83 \mathrm{~A}$. pisum genes (isolated from 8 scaffolds) with orthologues in the fruit fly genome and verified their localization (Figures 1-4).

In particular, in the scaffold 003383906 we identified 13 A. pisum genes with orthologues in fruit flies that mapped on Muller elements A, B, C, and D, but 8 of 13 mapped on the Muller element A (Figure 1).

The scaffold 003383512 presented 10 orthologous genes in A. pisum and D. melanogaster, and five of them mapped on the fruit fly Muller element $B$, whereas the other ones were located in elements $A$, C, and E (Figure 1).

The scaffold 003384156 contained only three orthologues and two of them mapped on Muller element E (Figure 2).

The scaffold 003383644 presented 12 orthologous genes and eight of them mapped on Muller element E, whereas the others mapped on D and C elements (Figure 2).

The scaffold 003383818 contained 13 orthologues and three of them mapped on Muller element $\mathrm{E}$, whereas the other ones mapped on A, B, C, and D elements (Figure 3).

The scaffold 003383768 contained 12 orthologous genes between aphids and flies, and six of them mapped on Muller element E, whereas the others mapped on A, C, and D elements (Figure 3). 
Scaffold 003383906

1 brain protein 44

2 pyruvate dehydrogenase

3 heat shock protein cognate 3

4 growth arrest-specific protein 2

5 UPF0518 protein

6 SMYD3

7 phosphatase 1

8 cell division protein 45

9 cisplatin associated protein

10 nucleoporin p58

11 GPN-loop GTPase

12 D2 cyclin

13 calcium channel

scaffold 003383906

scaffold 003383512

\section{Scaffold 003383512}

1 nup98

2 prefoldin subunit 3

3 small nuclear ribonucleoprotein $40 \mathrm{kDa}$

4 rho GTPase

5 sodium/potassium transporting ATPase

6 irregular chiasm $\mathrm{C}$ protein

7 kelch-like protein 2

8 exosomal core protein Cs14

9 Tret1

10 ribosomal protein S3

Figure 1. Gene content and reciprocal position of genes mapped in scaffold 003383906 and 003383512 in Acyrthosiphon pisum (green) and in Drosophila melanogaster (brown) Muller elements. 
Scaffold 003384156.1

1 coatomer subunit zeta- 1

2 dynein light chain 1

A

3 trifunctional purine biosyenthesis protein adenosie-3

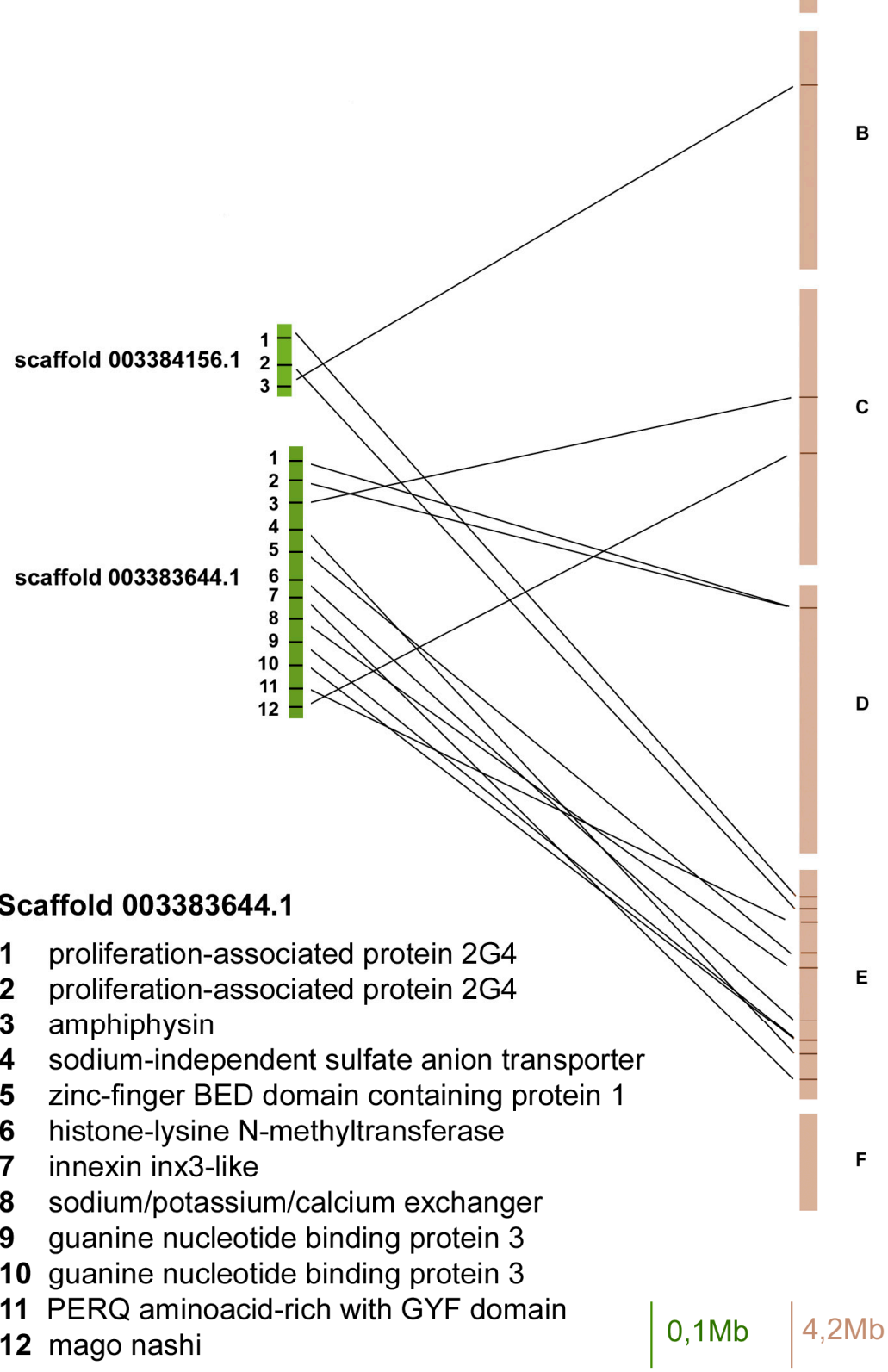

Figure 2. Gene content and reciprocal position of genes mapped in scaffold 003384156 and 003383644 in A. pisum (green) and in D. melanogaster (brown) Muller elements. 


\section{Scaffold 003383818}

1 piezo-type ion channel

2 beta-hexosaminidase subunit

3 STW domain protein

4 polypyrimidine tract binding protein

5 cytochrome P450

6 loss of heterozigosity homolog

7 chymotrypsinogen B

8 sodium-coupled transporter

9 gonadal protein gdl

10 regulator chromosome condensation

11 actin

12 trehalose synthase

13 GSK3 interacting protein

scaffold 003383818

scaffold 003383768

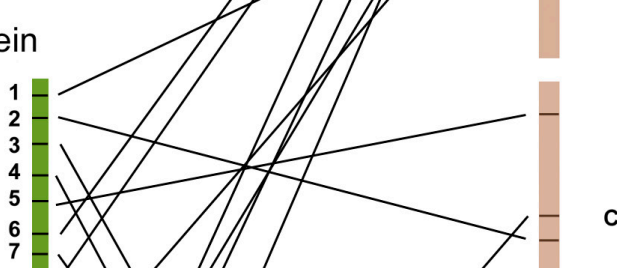

\section{Scaffold 003383768}

1 glucose dehydrogenase

2 NADH dehydrogenase

3 smoothelin-like protein 2

4 TAPT1

5 DARL anticodon-BP

6 vacuole membrane protein 1

7 Musashi homolog 2

8 beta-sarcoglycan

9 interleukin enhancer binding factor

10 vacuolar protein 62

11 cathepsin B

12 methyltransferase protein 16 homolog

Figure 3. Gene content and reciprocal position of genes mapped in scaffold 003383818 and 003383768 in A. pisum (green) and in D. melanogaster (brown) Muller elements.

Both the scaffold 003384165 and 003384041 contained 10 orthologous genes, but 9 out of 10 genes in the scaffold 003384165 mapped on Muller element $B$, whereas 6 out of 12 of the scaffold 003384041 have been located on element $\mathrm{E}$ (the other ones in Muller elements $\mathrm{A}, \mathrm{B}, \mathrm{C}$, and $\mathrm{D}$ ). 


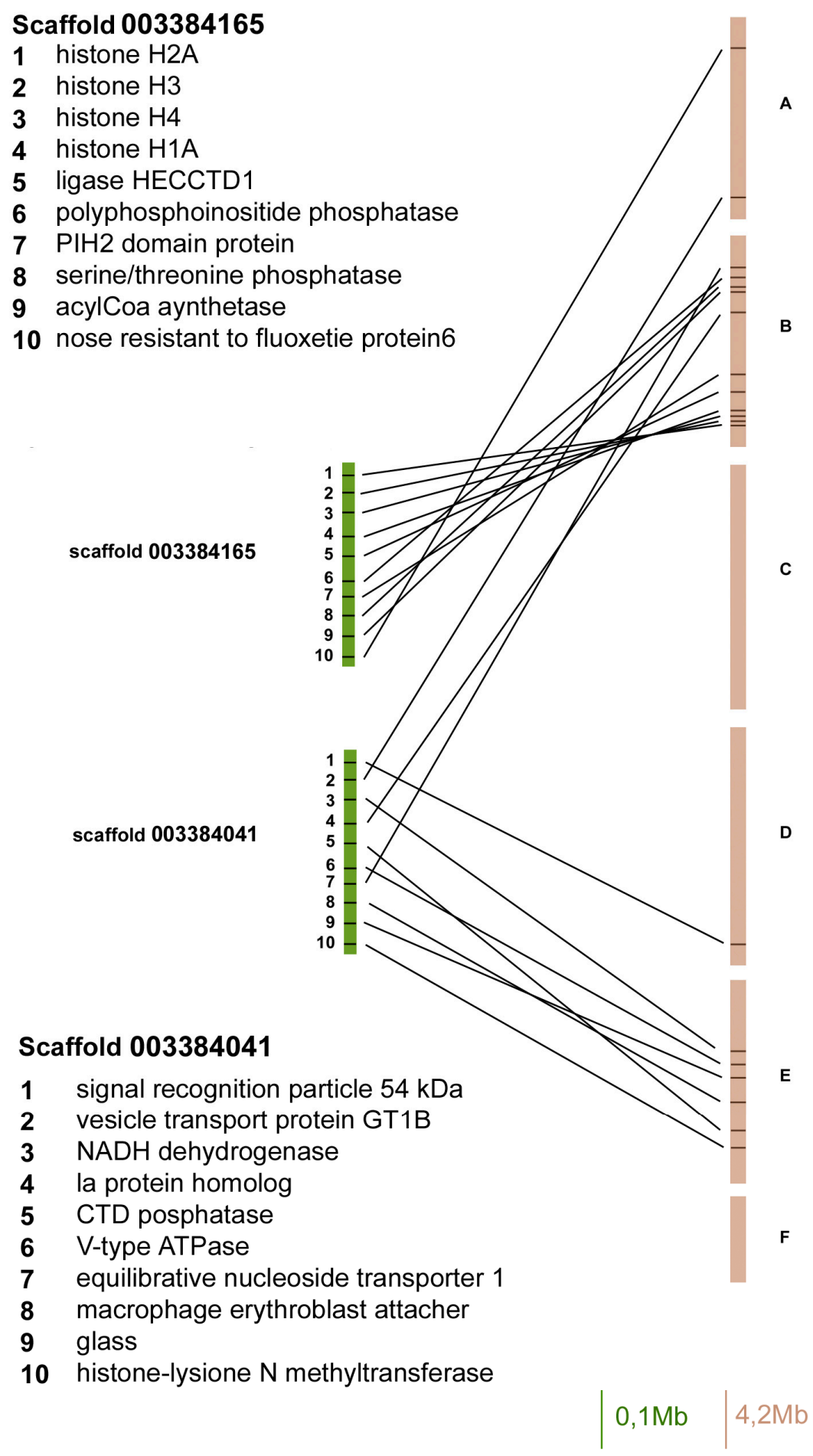

Figure 4. Gene content and reciprocal position of genes mapped in scaffold 003384165 and 003384041 in A. pisum (green) and in D. melanogaster (brown) Muller elements.

The chromosomal localization of the eight $A$. pisum scaffolds has been successively investigated by FISH. As summarized in Figure 5, the scaffolds 003383768 and 003383906 mapped on the opposite telomeres of the $\mathrm{X}$ chromosomes, identified since they are the unique ones with a chromomycin $\mathrm{A}_{3}$ $\left(\mathrm{CMA}_{3}\right)$ - fluorescent telomere, which is a rule in aphid complements (Figure 5a,c). Fluorescent in situ hybridization (FISH) experiments mapped scaffolds 003383512 and 003384165 in the same half 
of autosomes 1 (Figure 5a,d) and scaffolds 003384041, 003384156, and 003383644 on autosome 2 (Figure 5b,d). Scaffold 003383818 was the unique one mapping on autosome 3 (Figure 5c).

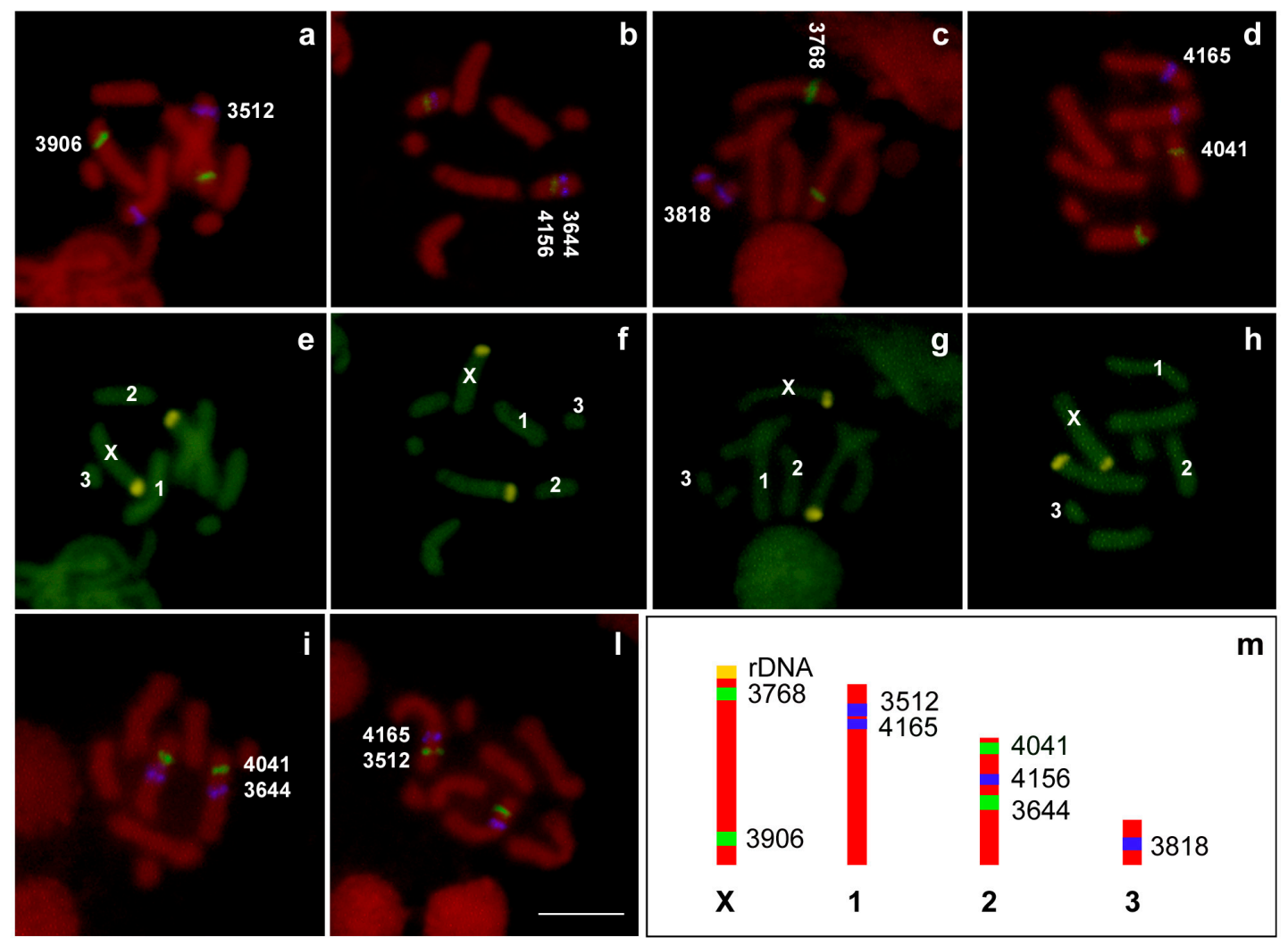

Figure 5. Double fluorescent in situ hybridization (FISH) with fluorescein isothiocyanate (FITC)-labelled 3906 (a), 4156 (b), 3768 (c), and 4041 (d) probes (in green) and with aminomethylcoumarin acetate (AMCA)-labelled 3512 (a), 3644 (b), 3818 (c), and 4165 (d) probes (in blue) allowed the mapping of the A. pisum scaffolds on the chromosomes, counterstained with propidium iodide (in red). In order to define the reciprocal position on chromosomes, double FISH experiments with FITC-labelled 4041 (i) and 3512 (1), and AMCA-labelled 3644 (i) and 4165 (1) probes have also been performed. Chromomycin $\mathrm{A}_{3}$ staining $(\mathbf{e}-\mathbf{h})$ allowed the identification of the $\mathrm{X}$ chromosomes in each plate. A schematic representation $(\mathbf{m})$ allowed the comparison of the localization of each scaffold on the A. pisum chromosomes. 3906: scaffold 003383906. 4156: scaffold 0033844156. 3768: scaffold 003383768. 4041: scaffold 003384041. 3512: scaffold 003383512. 3644: scaffold 003383644. 3818: scaffold 003383818. 4165: scaffold 003384165. Bar corresponds to $100 \mu \mathrm{m}$.

As a whole, the search of scaffolds with synteny between pea aphids and fruit flies allowed the identification of orthologous loci spanning all the four A. pisum chromosomes. Furthermore, our results show that, even if the gene order is highly scrambled, a trace of the Muller elements is present also in aphids (Figure 6). 


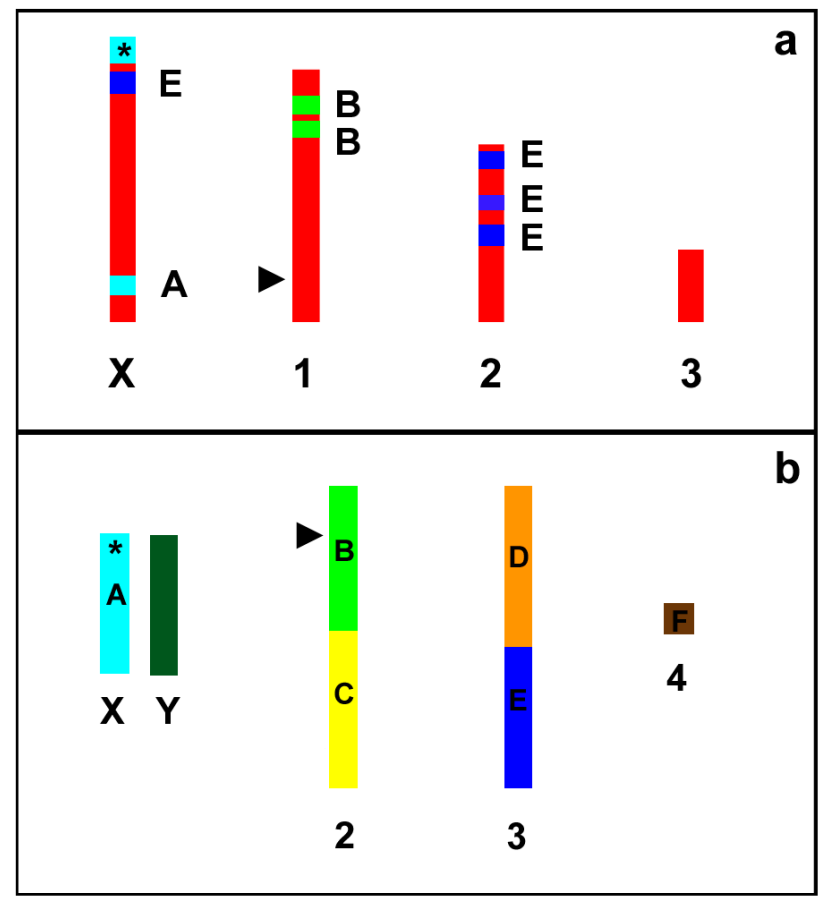

Figure 6. Schematic representation (not to scale) of the A. pisum (a) and D. melanogaster (b) karyograms showing the localization of the A-F Muller elements in the fly chromosomes and the trait of synteny with the Muller elements in the aphid chromosomes. Asterisks indicate the localization of the genes coding for ribosomal RNA (rDNA). Arrow heads indicate the position of the genes coding for 5S rRNA.

\section{Discussion}

D. melanogaster represents a largely utilized model system for animal and insect genetics [4,32-34]. The huge amount of available information from Drosophila provides valuable data for the analysis of gene regulation, genetic diseases, and evolutionary processes [4,32-34].

Several papers, published more than 70 years ago, evidenced the presence of recurrent traits of homology in the six chromosomal elements within the Drosophila genus [9,35]. The conservation of these basic elements, named A-F by Muller [35], has been successively confirmed also in the medfly Ceratitis capitata [36,37], as well as in the olive fly Bactrocera oleae [38]. Moreover, the chromosome homology among several Bactrocera species and C. capitate, as well as between Anastrepha ludens and C. capitate, has been established based on both their polytene chromosome banding pattern similarities and/or in situ hybridization of selected probes [15,39-43].

As reported by Sved et al. [15], Muller elements are not conserved in the Drosophila genus only, but also in tephritid fruit flies, even though they diverged from drosophilids about 60-70 mYr ago. According to data on Drosophila species, gene order may be highly scrambled within each Muller element, thus indicating high levels of intra-chromosomal rearrangements $[15,42,43]$. The stability in the gene chromosomal localization observed in Diptera is in stark contrast with data collected in other taxa [15]. Indeed, higher rates of inter-chromosomal rearrangements occurred over comparable time spans in most other groups, such as eutherian mammals [44], cichlid fishes [45], finches [46], and plants [47].

The presence and conservation of the Muller elements have not been studied outside Diptera, despite their potential usefulness to understand the evolution of the insect karyotype and the possibility to favour the development of chromosomal markers also in other insect species.

Although aphid chromosomes have been studied for several decades, at present few genes have been located on chromosomes, other than the 28S rDNA genes located at one telomere of the X chromosomes, and they include the 5S rDNA [24] and histone genes [48] in both A. pisum and 
M. persicae, as well as the esterase E4 coding genes in M. persicae only [49]. Moreover, ten satellite DNAs have been also identified and localized on chromosomes in five aphid species: one in Megoura viciae [50], one in Rhopalosiphon padi [51], two in M. persicae [22,52], two in Amphorophora tuberculata [53], and four in Aphis nerii [26].

In the present paper we localized more than 80 genes spanning all the four A. pisum chromosomes clearly supporting the proposal that the search for Muller elements could greatly improve the development of chromosome-specific markers in insects outside the Drosophila genus. Indeed, our results show that, even if the gene order is highly scrambled (as expected from the literature data reported about Diptera), a trace of the Muller elements is still present in aphids, even if the divergence between A. pisum and D. melanogaster can be estimated to 320-390 million years ago [54].

The presence of the trait of homologies/synteny between aphids and Diptera is particularly relevant considering that aphids possess holocentric chromosomes with kinetic activity spread along the whole chromosome axis, as well as a reproduction based on apomictic parthenogenesis that could favour the occurrence of chromosomal rearrangements and their inheritance [28,29].

The occurrence of this macrosynteny, combined to the presence of genes differently ordered and distributed on chromosomes, is similar to what was observed in the of holocentric chromosomes of Lepidoptera [55]. Indeed, as reported by d'Alençon et al. [53], a high degree of synteny was present between Bombyx mori and two noctuid species even if high rates of local genome rearrangements have been observed. Conserved syntenic blocks of genes were very small in Lepidoptera since they approximately contain 1.3 genes per block between B. mori and two noctuid species, and 2.0 genes per block between Spodoptera frugiperda and Helicoverpa armigera [55]. This corresponds to approximately two chromosome breaks per Mb DNA per million years, which is an evolution rate much higher than among species of the Drosophila genus [42,43]. It seems, therefore, that holocentric chromosomes could favour local rearrangement without significantly affecting the synteny at the overall chromosomal level.

A further element that could explain this result is related to the gene density and distribution on chromosomes $[28,29]$. Even if, at present, there is no detailed information regarding the distribution of genes on arthropod holocentric chromosomes [28,29], previous cytogenetic analyses suggested that, in aphids, the distribution of genes was uniform throughout all autosomes, with some differences related to $\mathrm{X}$ chromosomes where a certain degree of compartmentalization has been observed [56]. The occurrence of a uniform gene distribution counteracts data collected in other insects, such as D. melanogaste and Anopheles gambiae, where three isochore families have been identified with gene density increasing in GC-rich isochores [57] and could be a feature of species with holocentric chromosomes. As a whole, we can suggest that chromosome rearrangements, facilitated by the holocentric nature of chromosomes, disrupted gene-rich chromosomal regions, bringing them to a uniform gene distribution without affecting the overall macrosynteny among aphids and other insects.

Considering that aphids and Lepidoptera share the presence of holocentric chromosomes, it can be suggested that the scattered organization of centromeric determinants (related to their holocentric nature) may lead to a greater genomic plasticity, as chromosome fragments resulting from double-strand breaks can be maintained favouring intra-chromosomal rather than inter-chromosomal rearrangements.

According to literature data $[30,31]$, A pisum show substantial synteny of gene order and orientation with other Aphidinae, with excellent prospects for being able to extend genomic information from A. pisum to other aphid species. As a consequence, the study of Muller elements in aphids could favour the identification of the chromosomal marker also in other aphid species, such as M. persicae, where several chromosomal rearrangements have been observed, but a full molecular cytogenetics analysis is still lacking in view of the absence of chromosomal markers.

\section{Materials and Methods}

The specimens of the pea aphid, A. pisum, used in the present research, were obtained from the LSR1 laboratory lineage, kindly furnished by Manuel Plantagenest (INRA, Le Rheu, France) and 
maintained asexually on broad bean Vicia faba plants at $19{ }^{\circ} \mathrm{C}$ at a light-dark regime of $16 \mathrm{~h}$ light:8 $\mathrm{h}$ darkness. The A. pisum LSR1 lineage was used, since it is the pea aphid lineage sequenced for the genome project [16].

Chromosome preparations were obtained from parthenogenetic females by spreading embryo cells, as reported by Mandrioli et al. [22], whereas $\mathrm{CMA}_{3}$ staining was done as described by Mandrioli et al. [26].

DNA extraction was done using the Wizard ${ }^{\circledR}$ SV Genomic DNA Purification System (Promega, Madison, WI, USA), according to the manufacturer's instructions. The Long PCR Enzyme Mix (Fermentas, St. Leon-Rot, Germany), combined to a digoxigenin (DIG)- and biotin-labelling of the probe with the PCR DIG labelling kit (Roche, Sdney, Australia), has been used to amplify and label two contiguous $20 \mathrm{Kbp}$ long probes for each scaffold. The use of two probes for each scaffold has been preferred, since it allows longer labelled chromosomal portions and, consequently, more evident fluorescent signals on chromosomes. Oligonucleotide primers have been specifically designed on the scaffold sequences (Table 1) using the freely available software Primer 3 (available online: http://bioinfo.ut.ee/primer3/).

Table 1. List of primer for the amplification of the FISH probes.

\begin{tabular}{|c|c|c|}
\hline Scaffold/Probe Name & Primer Name & Primer Sequence \\
\hline \multirow{2}{*}{ Scaffold 0033844156 (NW_003384156) probe 1} & $4156 \mathrm{~F} 1$ & 5'-CTTGTATGTTTTGTATGCGTGAGAC-3' \\
\hline & 4156R1 & 5'-AACAAATTTCAGTTAAACGCAGAAC-3' \\
\hline \multirow{2}{*}{ Scaffold 0033844156 (NW_003384156) probe 2} & $4156 \mathrm{~F} 2$ & 5'-TATATGAATAAGCCATGACAAATAA-3' \\
\hline & $4156 \mathrm{R} 2$ & 5'-ATTATGAATATAAAGACGAGCCTAA-3' \\
\hline \multirow{2}{*}{ Scaffold 0033833644 (NW_003383644) probe 1} & 3644F1 & 5'-TAGGTAGCTGTATAACCCAGTTTCG-3' \\
\hline & 3644R1 & 5'-AACAGACGGTGTGTAGGTATGGTAT-3' \\
\hline \multirow{2}{*}{ Scaffold 0033833644 (NW_003383644) probe 2} & $3644 \mathrm{~F} 2$ & 5'-CAGCATTATACGCATAGGTAGGACT-3' \\
\hline & $3644 R 2$ & 5'-AAAACTTGTCATGTGTTTTCTGACA-3' \\
\hline \multirow{2}{*}{ Scaffold 003383818 (NW_003383818) probe 1} & 3818F1 & 5'-TTGTTCTCATTGGATTTATTTGGTT-3' \\
\hline & 3818R1 & 5'-AAGTGAGGTACTAATTCGTGTCCAG-3' \\
\hline \multirow{2}{*}{ Scaffold 0033833818 (NW_003383818) probe 2} & $3818 \mathrm{~F} 2$ & 5'-CTGGACACGAATTAGTACCTCACTT-3' \\
\hline & $3818 \mathrm{R} 2$ & 5'-TTCATTGCATACAAAACATGGTATC-3' \\
\hline \multirow{2}{*}{ Scaffold 003383768 (NW_003383768) probe 1} & 3768F1 & 5'-TACCAACGTCGTACATACACCATAC-3' \\
\hline & 3768R1 & 5'-ATTATTGATGCCCATTTTACTACGA-3' \\
\hline \multirow{2}{*}{ Scaffold 0033833768 (NW_003383768) probe 2} & $3768 \mathrm{~F} 2$ & 5'-TGGCTATGTGTCGTTATGAATTAGA-3' \\
\hline & $3768 \mathrm{R} 2$ & 5'-CCAAGTTTGTGAAAATGGTTAAATC-3' \\
\hline \multirow{2}{*}{ Scaffold 003383906 (NW_003383906) probe 1} & 3906F1 & 5'-TAGAAATCAGTGTCATGAAGGATGA-3' \\
\hline & 3906R1 & 5'-CTAGTCAACACGGGTAATGAGAGTT-3' \\
\hline \multirow{2}{*}{ Scaffold 0033833906 (NW_0033838906) probe 2} & $3906 \mathrm{~F} 2$ & 5'-ATCACTCACACATTCGTTTTCAGTA-3' \\
\hline & 3906R2 & 5'-TTATTTTCCACСАСТTTTCAATCAT-3' \\
\hline \multirow{2}{*}{ Scaffold 003383512 (NW_003383512) probe 1} & $3512 \mathrm{~F} 1$ & 5'-CGGTATCAGTTCGTTAAGCATAAGT-3' \\
\hline & 3512R1 & 5'-ATACAATTGATGAATCGGTTGAGTT-3' \\
\hline \multirow{2}{*}{ Scaffold 0033833512 (NW_0033838512) probe 2} & $3512 \mathrm{~F} 2$ & 5'-AACCAATACATTCAAGAATTTCCAA-3' \\
\hline & $3512 \mathrm{R} 2$ & 5'-CACACGACGTCATCTAGTACAAATC- $3^{\prime}$ \\
\hline \multirow{2}{*}{ Scaffold 003384165 (NW_003384165) probe 1} & $4165 \mathrm{~F} 1$ & 5'-TTTAATATTGATTGCTCCGTATGGT-3' \\
\hline & 4165R1 & 5'-TCATTATCCAAAAGAAAGGAGACTG-3' \\
\hline \multirow{2}{*}{ Scaffold 003384165 (NW_003384165) probe 2} & $4165 \mathrm{~F} 2$ & 5'-TGATACCGATTGTGATTTTAAGGAT-3' \\
\hline & 4165R2 & 5'-GTTCAAAGACTGATCGTACATGTTG-3' \\
\hline \multirow{2}{*}{ Scaffold 003384041 (NW_003384041) probe 1} & 4041F1 & 5'-TTGTACCTGCACATTGTAGACCTAA-3' \\
\hline & 4041R1 & 5'-ACAACTAACTGCAGGTCTTTATTGG-3' \\
\hline \multirow{2}{*}{ Scaffold 003384041 (NW_003384041) probe 2} & $4041 \mathrm{~F} 2$ & 5'-GATTTCTCATTGATACGGCTTCTAA-3' \\
\hline & 4041R2 & 5'-CCATGGTTTGAGTGTACTTCTTCTT-3' \\
\hline
\end{tabular}


Fluorescent in situ hybridization (FISH) was performed as described by Mandrioli et al. [26] using fluorescein isothiocyanate (FITC)-conjugated anti-DIG antibodies (Roche, Sidney, Australia) for the DIG labelled probes and aminomethylcoumarin acetate (AMCA) coniugated-avidin for the biotin labelled probes. FISH slides were observed using a Zeiss Axioplan epifluorescence microscope. Photographs of the fluorescent images were taken using a CCD camera (Spot, Digital Instrument, Madison, WI, USA) and the Spot software supplied with the camera and processed using Adobe Photoshop (Adobe Systems, Mountain View, CA, USA).

Bioinformatic analyses were done by BLAST alignments in Genbank (available online: http://blast.ncbi.nlm.nih.gov/Blast.cgi), both at DNA and protein level. Later, a further search was performed by BLAST alignments of aphid genomes using AphidBase (available online: http:/ / www.aphidbase.com). The assembly 2.0 of the pea aphid genome were used for our analyses.

Acknowledgments: We are indebted to Manuel Plantagenest (INRA, Le Rheu, France), who provided us with the A. pisum aphid lineage.

Author Contributions: Mauro Mandrioli and Gian Carlo Manicardi conceived and designed the experiments; Giada Zambonini and Mauro Mandrioli performed the experiments; Mauro Mandrioli and Gian Carlo Manicardi analysed the data and wrote the paper.

Conflicts of Interest: The Authors declare no conflict of interest.

\section{References}

1. Honeybee Genome Sequencing Consortium. Insights into social insects from the genome of the honey bee Apis mellifera. Nature 2006, 443, 931-949.

2. Tribolium Genome Sequencing Consortium. The genome of the model beetle and pest Tribolium castaneum. Nature 2008, 452, 949-955.

3. Brucker, R.M.; Funkhouser, L.J.; Setia, S.; Pauly, R.; Bordenstein, S.R. Insect innate immunity database (IIID): An annotation tool for identifying immune genes in insect genomes. PLoS ONE 2012, 7, e45125. [CrossRef] [PubMed]

4. Chilana, P.; Sharma, A.; Rai, A. Insect genomic resources: Status, availability and future. Curr. Sci. 2012, 102, 571-580.

5. Adams, M.D.; Celniker, S.E.; Holt, R.A.; Evans, C.A.; Gocayne, J.D.; Amanatides, P.G.; Scherer, S.E.; Li, P.W.; Hoskins, R.A.; Galle, R.F.; et al. The genome sequence of Drosophila melanogaster. Science 2000, 287, $2185-2195$. [CrossRef] [PubMed]

6. Tedeschi, R.; Monti, M.; Gonella, E.; Mandrioli, M. Molecular and cellular analysis of immunity in the phytoplasma vector Euscelidius variegatus: Exploiting immunity to improve biological control strategies. Invertebr. Surv. J. 2017, 14, 63-72.

7. Meller, V.H.; Kuroda, M.I. Sex and the single chromosome. Adv. Genet. 2002, 46, 1-24. [PubMed]

8. Landeen, E.L.; Presgraves, D.C. Evolution: From autosomes to sex chromosomes and back. Curr. Biol. 2013, 23, R848-R850. [CrossRef] [PubMed]

9. Sturtevant, A.H.; Novitski, E. The homologies of the chromosome elements in the genus Drosophila. Genetics 1941, 26, 517-541. [PubMed]

10. Zdobnov, E.; Mering, C.V.; Letunic, I.; Torrents, D.; Suyama, M.; Copley, R.R.; Christophides, G.K.; Thomasova, D.; Holt, R.A.; Subramanian, G.M.; et al. Comparative genome analysis of Anopheles gambiae and Drosophila melanogaster. Science 2002, 298, 149-159. [CrossRef] [PubMed]

11. Severson, D.W.; de Bruyn, B.; Lovin, D.D.; Brown, S.E.; Knudson, D.L.; Morlais, I. Comparative genome analysis of the yellow fever mosquito Aedes aegypti with Drosophila melanogaster and the malaria vector mosquito Anopheles gambiae. J. Hered. 2004, 95, 103-113. [CrossRef] [PubMed]

12. Bhutkar, A.; Schaeffer, S.W.; Russo, S.M.; Xu, M.; Smith, T.F.; Gelbart, W.M. Chromosomal rearrangement inferred from comparisons of 12 Drosophila genomes. Genetics 2008, 179, 1657-1680. [CrossRef] [PubMed]

13. Wallace, A.G.; Detweiler, D.; Schaeffer, S.W. Evolutionary history of the third chromosome gene arrangements of Drosophila pseudoobscura inferred from inversion breakpoints. Mol. Biol. Evol. 2011, 28, 2219-2229. [CrossRef] [PubMed] 
14. Vicoso, B.; Bachtrog, D. Reversal of an ancient sex chromosome to an autosome in Drosophila. Nature 2013, 499, 332-335. [CrossRef] [PubMed]

15. Sved, J.A.; Chen, Y.C.; Shearman, D.; Frommer, M.; Gichrist, A.S.; Sherwin, W.B. Extraordinary conservation of entire chromosomes in insects over long evolutionary periods. Evolution 2016, 70, 229-234. [CrossRef] [PubMed]

16. International Aphid Genomics Consortium. Genome sequence of the pea aphid Acyrthosiphon pisum. PLoS Biol. 2010, 8, e1000313.

17. Oerke, E.C. Estimated crop losses in wheat. In Crop Production and Crop Protection: Estimated Losses in Major Food and Cash Crops, 1st ed.; Elsevier: Amsterdam, The Netherland, 1994; pp. 179-296.

18. Blackman, R.L.; Eastop, V.F. Aphids on the World's Crops: An Identification and Information Guide, 2nd ed.; Wiley, John \& Sons, Incorporated: London, UK, 2000; p. 476.

19. Brisson, J.A.; Davis, G.K. Pea Aphid. In Genome Mapping and Genomics in Arthropods; Hunter, W., Kole, C., Eds.; Springer-Verlag: Berlin/Heidelberg, Germany, 2008; Volume 1, pp. 59-67.

20. Hawthorne, D.J.; Via, S. Genetic linkage of ecological specialization and reproductive isolation in pea aphids. Nature 2001, 412, 904-907. [CrossRef] [PubMed]

21. Braendle, C.; Caillaud, M.C.; Stern, D.L. Genetic mapping of aphicarus: A sex-linked locus controlling a wing polymorphism in the pea aphid (Acyrthosiphon pisum). Heredity 2005, 94, 435-442. [CrossRef] [PubMed]

22. Mandrioli, M.; Bizzaro, D.; Giusti, M.; Manicardi, G.C.; Bianchi, U. The role of rDNA genes in X chromosomes association in the aphid Acyrthosiphon pisum. Genome 1999, 42, 381-386. [CrossRef]

23. Mandrioli, M.; Bizzaro, D.; Manicardi, G.C.; Gionghi, D.; Bassoli, L.; Bianchi, U. Cytogenetic and molecular characterization of a highly repeated DNA sequence in the peach potato aphid Myzus persicae. Chromosoma 1999, 108, 436-442. [CrossRef]

24. Bizzaro, D.; Mandrioli, M.; Zanotti, M.; Giusti, M.; Manicardi, G.C. Chromosome analysis and molecular characterization of highly repeated DNAs in the aphid Acyrthosiphon pisum (Aphididae, Hemiptera). Genetica 2000, 108, 197-202.

25. Manicardi, G.C.; Mandrioli, M.; Bizzaro, D.; Bianchi, U. Cytogenetic and molecular analysis of heterochromatic areas in the holocentric chromosomes of different aphid species. In Chromosome Structure and Function; Sobti, R.G., Obe, G., Athwal, R.S., Eds.; Narosa Publishing House: New Delhi, India, 2002; pp. $47-56$.

26. Mandrioli, M.; Azzoni, P.; Lombardo, G.; Manicardi, G.C. Composition and epigenetic markers of heterochromatin in the aphid Aphis nerii (Hemiptera: Aphididae). Cytogenet. Genome Res. 2011, 133, 67-77. [CrossRef] [PubMed]

27. Mandrioli, M.; Manicardi, G.C. Unlocking holocentric chromosomes: New perspectives from comparative and functional genomics? Curr. Genom. 2012, 13, 343-349. [CrossRef] [PubMed]

28. Manicardi, G.C.; Mandrioli, M.; Blackman, R.L. The cytogenetic architecture of the aphid genome. Biol. Rev. 2015, 90, 112-125. [CrossRef] [PubMed]

29. Manicardi, G.C.; Nardelli, A.; Mandrioli, M. Fast chromosomal evolution and karyotype instability: Occurrence of recurrent chromosomal rearrangements in the peach potato aphid Myzus. persicae (Hemiptera, Aphididae). Biol. J. Linn. Soc. 2015, 116, 519-529. [CrossRef]

30. Moran, N.A.; Kaplan, M.E.; Gelsey, M.J.; Murphy, T.G.; Scholes, E.A. Phylogenetics and evolution of the aphid genus Uroleucon based on mitochondrial and nuclear DNA sequences. System Entomol. 1999, $24,85-93$. [CrossRef]

31. Von Dohlen, C.D.; TeulonM, D.A.J. Phylogeny and historical biogeography of New Zealand indigenous Aphidini aphids (Hemiptera, Aphididae): An hypothesis. Ann. Entomol. Soc. Am. 2003, 96, 107-116. [CrossRef]

32. Schneider, D. Using Drosophila as a model insect. Nat. Rev. Genet. 2000, 1, 218-226. [CrossRef] [PubMed]

33. Arias, A.M. Drosophila melanogaster and the development of biology in the 20th century. Methods Mol. Biol. 2008, 420, 1-25. [PubMed]

34. Ashburner, M.; Bergman, C.M. Drosophila melanogaster: A case study of a model genomic sequence and its consequence. Genome Res. 2005, 15, 1661-1667. [CrossRef] [PubMed]

35. Muller, H.J. Bearings of the Drosophila work on systematics. In The New Systematics; Huxley, J., Ed.; Oxford University Press: Oxford, UK, 1940; pp. 185-268. 
36. Zacharopoulou, A.; Frisardi, M.; Savakis, C.; Robinson, A.S.; Tolias, P.; Konsolaki, M. The genome of the Mediterranean fruitfly Ceratitis capitata: Localization of molecular markers by in situ hybridization to salivary gland polytene chromosomes. Chromosoma 1992, 101, 448-455. [CrossRef] [PubMed]

37. Gariou-Papalexiou, A.; Gourzi, P.; Delprat, A.; Kritikou, D.; Rapti, K.; Chrysanthakopoulou, B. Polytene chromosomes as tools in the genetic analysis of the Mediterranean fruit fly, Ceratitis capitata. Genetica 2002, 116, 59-71. [CrossRef] [PubMed]

38. Tsoumani, K.T.; Augustinos, A.A.; Kakani, E.G.; Drosopoulou, E.; Mavragani-Tsipidou, P.; Mathiopoulos, K.D. Isolation, annotation and applications of expressed sequence tags from the olive fly, Bactrocera oleae. Mol. Genet. Genom. 2011, 285, 33-45. [CrossRef] [PubMed]

39. Mavragani-Tsipidou, P. Genetic and cytogenetic analysis of the olive fruit fly Bactrocera oleae (Diptera: Tephritidae). Genetica 2002, 116, 45-57. [CrossRef] [PubMed]

40. Drosopoulou, E.; Nakou, I.; Mavragani-Tsipidou, P. The Bactrocera. Oleae genome: Localization of nine genes on the polytene chromosomes of the olive fruit fly (Diptera: Tephritidae). Genome 2014, 57, 573-576. [CrossRef] [PubMed]

41. Augustinos, A.A.; Drosopoulou, E.; Gariou-Papalexiou, A.; Asimakis, E.D.; Cáceres, C.; Tsiamis, G. Cytogenetic and symbiont analysis of five members of the B. dorsalis complex (Diptera, Tephritidae): No evidence of chromosomal or symbiont-based speciation events. Zookeys 2015, 540, 273-298.

42. Orengo, D.J.; Puerma, E.; Papaceit, M.; Segarra, C.; Aguadé, M. Dense gene physical maps of the non-model species Drosophila subobscura. Chromosome Res. 2017, 25, 145-154. [CrossRef] [PubMed]

43. Richards, S.; Liu, Y.; Bettencourt, B.R.; Hradecky, P.; Letovsky, S.; Nielsen, R.; Thornton, K.; Hubisz, M.J.; Chen, R.; Meisel, R.P. Comparative genome sequencing of Drosophila pseudoobscura: Chromosomal, gene, and cis-element evolution. Genome Res. 2005, 15, 1-18. [CrossRef] [PubMed]

44. Foote, M.; Hunter, J.P.; Janis, C.M.; Sepkoski, J.J. Evolutionary and preservational constraints on origins of biologic groups: Divergence times of eutherian mammals. Science 1999, 283, 1310-1314. [CrossRef] [PubMed]

45. Poletto, A.B.; Ferreira, I.A.; Cabral-de-Mello, D.C.; Nakajima, R.T.; Mazzuchelli, J.; Ribeiro, H.B.; Venere, P.C.; Nirchio, M.; Kocher, T.D.; Martins, C. Chromosome differentiation patterns during cichlid fish evolution. BMC Genet. 2010, 11, 50-61. [CrossRef] [PubMed]

46. Hooper, D.M.; Price, T.D. Rates of karyotypic evolution in Estrildid finches differ between island and continental clades. Evolution 2015, 69, 890-903. [CrossRef] [PubMed]

47. Darlington, C.D.; Wylie, A.P. Chromosome atlas of flowering plants, 2nd ed.; Allen \& Unwin: London, UK, 1955.

48. Mandrioli, M.; Manicardi, G.C. Chromosomal mapping reveals a dynamic organization of the histone genes in aphids (Hemiptera: Aphididae). Entomologia 2013, 1, 1. [CrossRef]

49. Blackman, R.L.; Spence, J.M.; Field, L.M.; Devonshire, A.L. Chromosomal localization of the amplified esterase genes conferring resistance to insecticides in the aphid Myzus persicae. Heredity 1995, 75, 297-302. [CrossRef]

50. Bizzaro, D.; Manicardi, G.C.; Bianchi, U. Chromosomal localization of a highly repeated EcoRI DNA fragment in Megoura viciae (Homoptera, Aphididae) by nick translation and FISH. Chrom. Res. 1996, 4, 392-396. [CrossRef] [PubMed]

51. Monti, V.; Manicardi, G.C.; Mandrioli, M. Distribution and molecular composition of heterochromatin in the holocentric chromosomes of the aphid Rhopalosiphum padi (Hemiptera: Aphididae). Genetica 2010, 138, 1077-1084. [CrossRef] [PubMed]

52. Spence, J.M.; Blackman, R.L.; Testa, J.M.; Ready, P.D. A 169 bp tandem repeat DNA marker for subtelomeric heterochromatin and chromosomal re-arrangement in aphids of the Myzus persicae group. Chrom. Res. 1998, 6, 167-175. [CrossRef] [PubMed]

53. Spence, J.M.; Blackman, R.L. Orientation of the stretched univalent $X$ chromosome during the unequal first meiotic division in male aphids. Chrom. Res. 1998, 6, 177-181. [CrossRef] [PubMed]

54. Hedges, S.B.; Marin, J.; Suleski, M.; Paymer, M.; Kumar, S. Tree of life reveals clock-like speciation and diversification. Mol. Biol. Evol. 2015, 32, 835-845. [CrossRef] [PubMed]

55. D’Alençon, E.; Sezutsu, H.; Legeai, F.; Permal, E.; Bernard-Samain, S.; Gimenez, S.; Gagneur, C.; Cousserans, F.; Shimomura, M.; Brun-Barale, A.; et al. Extensive synteny conservation of holocentric chromosomes in Lepidoptera despite high rates of local genome rearrangements. Proc. Natl. Acad. Sci. USA 2010, 107, 7680-7685. [CrossRef] [PubMed] 
56. Manicardi, G.C.; Mandrioli, M.; Bizzaro, D.; Bianchi, U. Patterns of DNase I sensitivity in the holocentric chromosomes of the aphid Megoura viciae. Genome 1998, 41, 169-172. [CrossRef]

57. Cammarano, R.; Costantini, M.; Bernardi, G. The isochore patterns of invertebrate genomes. BMC Genom. 2009, 10, 538. [CrossRef] [PubMed] 